\title{
Risk factors related to the recurrence of pneumothorax in patients with emphysema
}

\author{
Beomsu Shin ${ }^{1}$, Sae Byol Kim¹, Chang Wan Kim², Il Hwan Park², Won-Yeon Lee ${ }^{1}$, Chun Sung Byun ${ }^{2}$ \\ ${ }^{1}$ Department of Internal Medicine, ${ }^{2}$ Department of Thoracic and Cardiovascular Surgery, Wonju College of Medicine, Yonsei University, Wonju-si, \\ Gangwon-do, Republic of Korea \\ Contributions: (I) Conception and design: B Shin, CS Byun; (II) Administrative support: WY Lee; (III) Provision of study materials or patients: B \\ Shin, IH Park, CS Byun; (IV) Collection and assembly of data: SB Kim, CW Kim, IH Park, WY Lee; (V) Data analysis and interpretation: B Shin, \\ SB Kim, CW Kim, CS Byun; (VI) Manuscript writing: All authors; (VII) Final approval of manuscript: All authors. \\ Correspondence to: Chun Sung Byun, MD, PhD. Department of Thoracic and Cardiovascular Surgery, Yonsei University Wonju College of Medicine, \\ 20 Ilsan-ro, Wonju-si, Gangwon-do, 26426, Republic of Korea. Email: csbyun@yonsei.ac.kr.
}

Background: Pneumothorax refers to the abnormal presence of air in the thoracic cavity. Pulmonary emphysema (PE) is often detected during computed tomography (CT), one of the radiological investigations used to diagnose pneumothorax and devise treatment plans in former or current smokers who present with pneumothorax. However, there are few reports that describe the recurrence rate and risk factors associated with recurrence in patients with PE and pneumothorax.

Methods: This study retrospectively cross-sectional analyzed the medical records of 164 patients diagnosed with their first episode of secondary spontaneous pneumothorax and admitted to a tertiary care hospital, between March 2013 and February 2019. The CT scans of 98 patients revealed PE, and 49 patients of those underwent pulmonary function tests (PFTs) after the resolution of pneumothorax. Risk factors for recurrence were analyzed using Cox proportional hazard regression.

Results: All the subjects were male and former or current smokers, with a median age of 72 years. Thirty-seven (75.5\%) patients were treated with tube thoracostomy alone, whereas 12 (24.5\%) patients underwent chemical pleurodesis via chest tube or video-assisted thoracoscopic surgery. After recovery from pneumothorax, these patients underwent PFT within a median time period of 5.8 months. Median forced vital capacity and forced expiratory volume in 1 second $\left(\mathrm{FEV}_{1}\right)$ were $3.02 \mathrm{~L}$ (91\% predicted) and $1.58 \mathrm{~L}$ (67\% predicted), respectively. In the current study, the recurrence rate of pneumothorax was observed to be $30.6 \%$, within a median time period of 12.4 months. In multivariable-adjusted analysis, decreased $\mathrm{FEV}_{1}$ was observed to be significantly higher in the patients who exhibited recurrence of pneumothorax than in those who did not (adjusted hazard ratio, 0.408; $\mathrm{P}=0.025$ ).

Conclusions: In patients with PE and pneumothorax, PFT, performed after recovery, could be a useful test for predicting the recurrence of pneumothorax.

Keywords: Pulmonary emphysema (PE); pneumothorax; recurrence; respiratory function tests

Submitted Apr 15, 2020. Accepted for publication Aug 21, 2020.

doi: $10.21037 /$ jtd-20-1557B

View this article at: http://dx.doi.org/10.21037/jtd-20-1557B

\section{Introduction}

Pneumothorax is defined as the abnormal presence of air in the pleural space that causes symptoms such as breathlessness or pleuritic chest pain $(1,2)$. Spontaneous pneumothorax, occurring spontaneously without any preceding trauma or injury, is further divided into primary spontaneous pneumothorax (PSP) and secondary spontaneous pneumothorax (SSP) (3). SSP accounts for about $20 \%$ of all pneumothorax and usually develops in 
elderly patients with structural lung disease $(4,5)$.

The most common underlying structural lung disease, which leads to SSP, is chronic obstructive pulmonary disease (COPD), followed by interstitial lung disease (ILD), malignancy, tuberculosis, cystic fibrosis, and necrotizing pneumonia (6-8). Compared with PSP, SSP is more closely associated with higher morbidity, mortality, and recurrence rates, because of the aforementioned advanced age and underlying structural lung diseases $(9,10)$. Aggressive treatment and prevention of recurrence are important for these reasons (11). Most patients with SSP undergo tube thoracostomy and some undergo additional chemical pleurodesis to stop the air leakage and prevent recurrent pneumothorax (4). In case of persistent air leakage, surgical treatment may be considered in some patients. However, in those with underlying lung disease and suboptimal pulmonary function, surgery is usually avoided at the first episode of pneumothorax (12).

COPD, the most common etiology of SSP, is a heterogeneous syndrome characterized by airflow limitation, as determined by the pulmonary function test (PFT), which is usually caused by the inhalation of cigarette smoke or other noxious particles $(13,14)$. Pulmonary emphysema (PE), one of the components of COPD, is defined as the permanent enlargement of air spaces accompanied by the destruction of gas-exchanging surfaces of the lung (15). Previously, PE was assessed through histological examinations. Advances in imaging technology have made it possible to evaluate $\mathrm{PE}$ through computed tomography (CT). As a result, PE have been described in CT images and include subtypes such as centrilobular, panlobular, and paraseptal emphysema (16).

PE can make patients more susceptible to pneumothorax and is one of the most commonly observed radiographic finding in the CT scans of patients with $\operatorname{SSP}(17,18)$. Nevertheless, few studies have evaluated the recurrence rate, risk factors associated with recurrence, and the outcomes of the treatment of pneumothorax in patients with PE $(19,20)$. Severe COPD with $\mathrm{FEV}_{1}<1 \mathrm{~L}$ has been reported to be associated with an increased risk of SSP (3). However, PE without airflow limitation was observed in $17 \%$ of the subjects in a previous study (21). Therefore, this study aimed to investigate the recurrence rate, treatment outcomes, and risk factors related to the recurrence of pneumothorax, diagnosed through CT scans, in patients with PE. We present the following article in accordance with the STROBE reporting checklist (available at http:// dx.doi.org/10.21037/jtd-20-1557B).

\section{Methods}

\section{Study population}

The study was conducted in accordance with the Declaration of Helsinki (as revised in 2013). The study was approved by the Institutional Review Board for Human Research of Yonsei University Wonju Severance Christian Hospital (CR-319180). Informed consent was waived of the retrospective nature of the research. Also, only de-identified data extracted from medical records prior to analysis were used in the study.

The current study involved a retrospective review of the medical records of all patients who presented with spontaneous pneumothorax at Wonju Severance Christian Hospital, a tertiary care hospital in South Korea, from March 2013 to February 2019. The records of 164 patients, who presented with SSP in the emergency department of our institution, were reviewed. Patients with pneumoconiosis $(n=24)$, pulmonary tuberculosis or non-tuberculous mycobacterial disease $(n=15)$, pneumonia $(\mathrm{n}=13)$, ILD $(\mathrm{n}=11)$, malignancy $(\mathrm{n}=2)$, or bronchiectasis $(n=1)$ were excluded from the study, resulting in 98 patients with PE. Additionally, 49 patients who did not undergo PFT after complete resolution of pneumothorax were excluded from the study. Consequently, 49 patients were included in the current study (Figure 1).

\section{Data collection}

The present study reviewed the following characteristics of each patient: age, sex, weight, height, body mass index (BMI), history of tobacco smoking, laboratory data, chest CT images used for the diagnosis of pneumothorax, previous history of pneumothorax, and treatment methods received to resolve the pneumothorax. PFT was performed after the recovery from pneumothorax, which was considered to be resolved after the removal of chest tube, along with the observation of radiographic evidence for pneumothorax resolution on a chest $\mathrm{X}$-ray. Additionally, the current study evaluated the recurrence rates of ipsilateral and contralateral pneumothorax. In the present study, the recurrence of pneumothorax was defined as the emergence of a pneumothorax after discharge, which was the primary cause of re-admission and subsequently, required management (20).

\section{Image analysis}

Abnormal CT findings such as centrilobular or paraseptal 


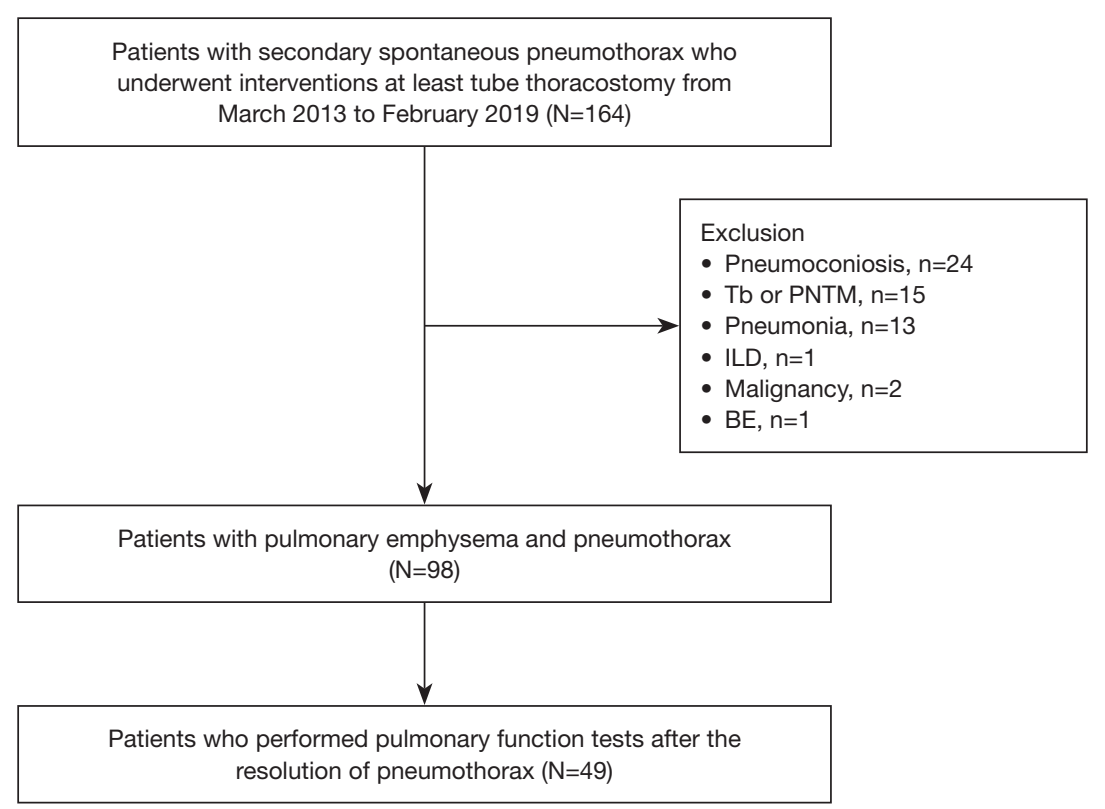

Figure 1 Flow chart of the study population. Tb, tuberculosis; PNTM, pulmonary non-tuberculous mycobacterial disease; ILD, interstitial lung disease; BE, bronchiectasis.

emphysema were interpreted in accordance with the Fleischner Society criteria. Centrilobular emphysema was defined as a centrilobular area of decreased attenuation without visible walls and paraseptal emphysema was defined as a sub pleural and peri-bronchovascular region of low attenuation separated by intact interlobular septa (16).

\section{Management of pneumothorax}

After hospital admission, a 20- or 24-French chest tube was inserted under local anesthesia. As per the established guidelines, air leakage lasting for more than 5 days was considered as prolonged air leakage $(11,22)$. In these cases of prolonged air leakage, the management involved either chemical pleurodesis [extract of Viscum album (Abnoba Viscum, Abnoba GmbH, Germany)] via chest tube on bedside or video-assisted thoracoscopic surgery (VATS); as determined by an attending physician, who consulted a respiratory physician and a thoracic surgeon, and based on the patient's conditions such as cardiopulmonary reservoir and past medical history.

\section{PFT}

Spirometry was performed using a Vmax 22 system (CareFusion, Yorba Linda, CA, USA) according to provisions discussed by the American Thoracic Society/ European Respiratory Society (23). Absolute values of the volume of air forcibly exhaled from the point of maximal inspiration (forced vital capacity, FVC) and the volume of air exhaled during the first second of the method (forced expiratory volume in 1 second, $\mathrm{FEV}_{1}$ ) were obtained and the ratio of the predicted values of $\mathrm{FEV}_{1}$ and $\mathrm{FVC}$ were calculated using a formula obtained from a the reference values, based on age, sex, race, and height $(24,25)$.

\section{Statistical analysis}

The data obtained are presented as either numbers (\%) or medians [interquartile range (IQR)]. The Mann-Whitney $\mathrm{U}$-test was used to compare continuous variables. The Kaplan-Meier method was used to estimate the recurrence rates after the primary episode of pneumothorax. A multivariable Cox proportional hazard regression analysis with backward stepwise selection, with $\mathrm{P}<0.05$ for the entry of variables and $\mathrm{P}>0.10$ for the removal of variables, was used to identify prognostic factors associated with recurrence. Results obtained are presented as hazard ratio (HR) with $95 \%$ confidence interval (CI). Statistical differences were considered significant at $\mathrm{P}<0.05$. All statistical analyses were performed using SPSS software version 23.0 (IBM Co., Chicago, IL, USA). 


\section{Results}

\section{Patient characteristics}

The clinical characteristics of the patients involved in the present study are presented in Table 1 . All the patients were male. The median age was 72 years, and $32(65.3 \%)$ patients were above the age of 65 years. The median height and BMI were $164 \mathrm{~cm}$ and $21.1 \mathrm{~kg} / \mathrm{m}^{2}$, respectively. Moreover, all the patients were former or current smokers. The median serum level of albumin was $4.1 \mathrm{~g} / \mathrm{dL}$ and the median partial pressure of carbon dioxide $\left(\mathrm{CO}_{2}\right)$ was $33 \mathrm{mmHg}$. The most common type of emphysema observed was the centrilobular type $(\mathrm{n}=23,46.9 \%)$. Regarding the affected side, 28 (57.1\%) and $21(42.9 \%)$ cases were affected by pneumothorax of the right and left side of the thoracic cavity, respectively. Cases that developed pneumothorax on both sides of the thoracic cavity were not detected in the present study.

\section{Treatment modalities and outcomes}

The treatment modalities and outcomes are presented in Table 2. Tube thoracostomy was performed in all the patients and was observed to be successful in 37 (75.5\%) patients. Either chemical pleurodesis via chest tube or VATS was performed in the $12(24.5 \%)$ patients with prolonged air leak. Although chemical pleurodesis via chest tube or VATS were sufficient treatment 9 of the 12 patients, 3 patients required additional chemical pleurodesis after VATS with pleurodesis. The median length of hospital stay was 12 days.

\section{Lung function tests}

The patients underwent PFT within a median time period of 5.8 months after the recovery from pneumothorax and the results are presented in Table 3. The median FVC and $\mathrm{FEV}_{1}$ were observed to be $3.02 \mathrm{~L}$ (91\% predicted) and $1.58 \mathrm{~L}$ (67\% predicted), respectively. In the patients who experienced recurrence of pneumothorax, the median FVC and $\mathrm{FEV}_{1}$ were observed to be $2.97 \mathrm{~L}$ ( $90 \%$ predicted) and $1.20 \mathrm{~L}$ (59\% predicted), respectively. Moreover, the median FVC and $\mathrm{FEV}_{1}$ were observed to be $2.85 \mathrm{~L}$ (89\% predicted) and $1.05 \mathrm{~L}$ (55\% predicted), respectively, in patients with ipsilateral recurrence of pneumothorax and $3.11 \mathrm{~L}(95 \%$ predicted) and $2.01 \mathrm{~L}$ (97\% predicted), respectively, in patients with contralateral recurrence of pneumothorax. The data is available in a supplementary appendix online and is presented in Table S1.
Table 1 Baseline characteristics

\begin{tabular}{lc}
\hline Variables & Value $(\mathrm{n}=49)$ \\
\hline Age, years & $72[63-76]$ \\
Sex, male & $49(100.0)$ \\
Body mass index, $\mathrm{kg} / \mathrm{m}^{2}$ & $21.1(19.1-24.1)$ \\
Height, cm & $164[159-170]$ \\
Smoking history & \\
Former smoker & $40(81.6)$ \\
Current smoker & $9(18.4)$ \\
Laboratory findings & \\
White blood cell, / $\mathrm{LL}$ & $8,790(7,255-12,765)$ \\
C-reactive protein, mg/dL & $0.44(0.29-1.62)$ \\
Albumin, g/dL & $4.1(3.8-4.4)$ \\
Partial pressure of carbon dioxide, & $33[29-42$ \\
mmHg & $23(24.6)$ \\
Observation period, months & \\
Type of emphysema on computed & \\
tomography & \\
Centrilobular & \\
Paraseptal & \\
pombined centrilobular and & \\
Paraptal & \\
\hline
\end{tabular}

Data are presented as number (\%) or the median (interquartile range).

Twelve (24.5\%) patients showed $\mathrm{FEV}_{1} / \mathrm{FVC}<0.7,3$ of whom were in the recurrence group.

\section{Recurrence and risk factors}

During the median observation period of 24 months, the overall recurrence rate after treatment was $30.6 \%$, affecting 14 patients who underwent tube thoracostomy and 1 patient who underwent tube thoracostomy and chemical pleurodesis. On the contrary, in the current study, recurrence of pneumothorax was not observed in the patients who underwent VATS with pleurodesis. Among the 15 patients that presented with recurrence, 8 presented with ipsilateral recurrence and 7 presented with contralateral recurrence (Figure 2). The more detailed data is available in a supplementary appendix online and is shown in Table $S 2$.

Univariable and multivariable Cox proportional hazard regressions were performed to evaluate the prognostic 
factors related to recurrence (Table 4). In univariable analysis, partial pressure of $\mathrm{CO}_{2}$ (unadjusted $\mathrm{HR}$ 1.041; $\mathrm{P}=0.040$ ) and $\mathrm{FEV}_{1}$ (unadjusted HR 0.477; $\mathrm{P}=0.049$ ) were observed to be significantly associated with pneumothorax recurrence. Multivariable analysis revealed that decreased $\mathrm{FEV}_{1}$ (adjusted HR 0.408; $\mathrm{P}=0.025$ ) showed a positive association with recurrence, whereas additional treatments such as chemical pleurodesis or VATS were not observed to

Table 2 Treatment modalities and outcomes

\begin{tabular}{lc}
\hline Variables & Value $(\mathrm{n}=49)$ \\
\hline Affected side & $21(42.9)$ \\
Left & $28(57.1)$ \\
Right & \\
Treatments & $37(75.5)$ \\
Tube thoracostomy & $6(12.2)$ \\
$\begin{array}{l}\text { Tube thoracostomy with chemical } \\
\text { pleurodesis }\end{array}$ & $6(12.2)$ \\
$\quad$ Video-assisted thoracoscopic surgery with \\
chemical pleurodesis
\end{tabular}

Data are presented as number (\%) or the median (interquartile range). be significantly associated with recurrence, compared with tube thoracostomy alone (adjusted HR 3.427; $\mathrm{P}=0.241$ ).

\section{Discussion}

In patients with SSP, the reported recurrence rate of pneumothorax is approximately $26 \%$ to $50 \%$ (10). While treating SSP, the prevention of recurrence of pneumothorax needs to be considered. However, there are no known risk factors related to the recurrence of SSP. In the present study, the overall recurrence rate, after the treatment of patients with $\mathrm{PE}$ and pneumothorax, was $30.6 \%$ and decreased $\mathrm{FEV}_{1}$ was observed to be a risk factor related to the recurrence of pneumothorax.

Pneumothorax causes chronic morbidity in patients with chronic lung disease worldwide $(26,27)$. A recent study showed that 14 patients per 100,000 populations in the United States were hospitalized due to pneumothorax in the year 2016; among which, $61 \%$ of patients had chronic lung disease, a majority were elderly and had PE (7). In the present study, the median age of patients was 72 years and all the patients exhibited PE.

Patients suffering from SSP can be treated by means of various methods $(11,22)$. Nevertheless, pulmonary function usually gets compromised and there may be many obstacles related to treatments such as surgery $(4,20)$. Adverse events related to chemical pleurodesis should also be considered $(2,28)$. Although tube thoracostomy is a frequently employed of treatment, the best treatment approach is still controversial. In the present study, 14 patients (14/37; $37.8 \%)$ treated with tube thoracostomy alone presented with recurrent pneumothorax, whereas only one patient $(1 / 6 ; 16.7 \%)$ treated with chemical pleurodesis via chest

Table 3 Pulmonary function tests after recovery from pneumothorax

\begin{tabular}{|c|c|c|c|c|}
\hline Variables & $\mathrm{N}=49$ & \multicolumn{3}{|c|}{ Recurrence } \\
\hline FVC, L & $3.02(2.64-3.47)$ & $2.97(2.56-3.31)$ & $3.08(2.65-3.76)$ & 0.260 \\
\hline FVC, \% predicted & 91 [76-100] & 90 [74-96] & 92 [79-102] & 0.304 \\
\hline $\mathrm{FEV}_{1}, \mathrm{~L}$ & $1.58(1.06-2.19)$ & $1.20(0.91-2.01)$ & $1.77(1.23-2.29)$ & 0.165 \\
\hline $\mathrm{FEV}_{1} / \mathrm{FVC}$ & $0.53(0.37-0.70)$ & $0.51(0.32-0.65)$ & $0.57(0.38-0.71)$ & 0.306 \\
\hline $\mathrm{FEV}_{1} / \mathrm{FVC}<0.7$ & $37(75.5)$ & $12(80.0)$ & $25(73.5)$ & - \\
\hline
\end{tabular}

Data are presented as number (\%) or the median (interquartile range). FVC, forced vital capacity; FEV ${ }_{1}$, forced expiratory volume in 1 second. 


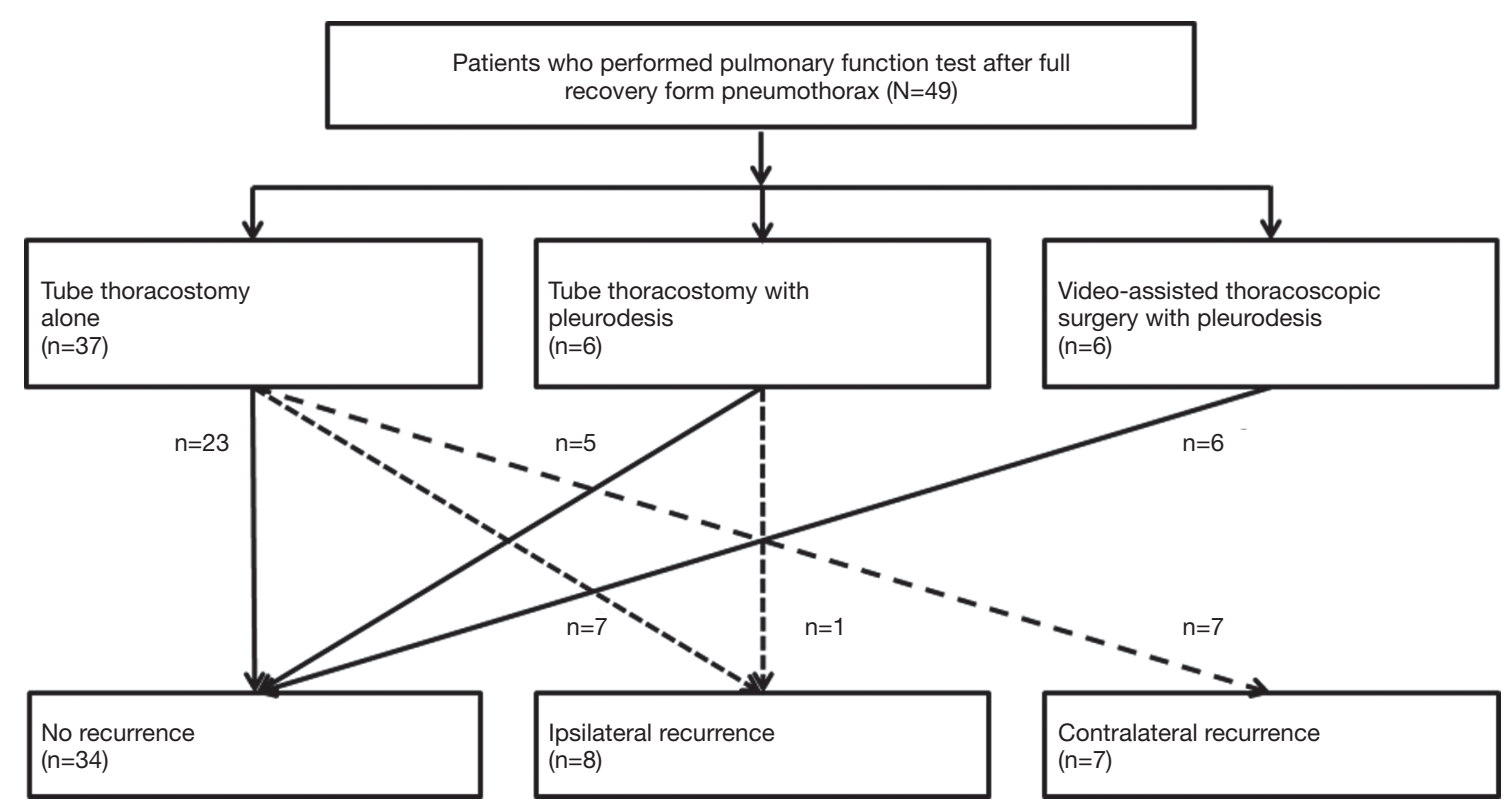

Figure 2 Management of pneumothorax and treatment outcomes at the study population.

tube presented with recurrent pneumothorax and no recurrence of pneumothorax was observed in the six patients treated with VATS with pleurodesis. Patients treated with tube thoracostomy alone were observed to have a tendency towards higher rates of recurrence, compared to those who underwent tube thoracostomy with pleurodesis or VATS with pleurodesis, although the difference was not observed to be statistically significant in the present study $(\mathrm{P}=0.241)$.

It is an established fact that tube thoracostomy with pleurodesis or VATS with pleurodesis cannot be performed in all patients, in order to reduce the recurrence rate of pneumothorax; considering the general condition of patients with diminished pulmonary reserve. Hence, followup of the patients who underwent suboptimal treatment is very important. However, no standardized strategies have been established regarding the observation protocols and periodic examinations for the prevention of recurrent pneumothorax in such patients (8). According to the previous guidelines, patients with SSP should be followed up by a respiratory physician to evaluate and manage the underlying lung disease (11). However, this is not followed in actual clinical practice, in which many patients with SSP do not undergo regular follow-up by a respiratory physician or PFT. The present study observed that 49 among the 98 patients did not undergo PFT, after the complete resolution of pneumothorax. Some of the patients were referred back to their primary medical institutions after discharge and others had improved respiratory symptoms and refused further evaluation of the underlying lung disease (29).

Spirometry is one of the tools used in the evaluation of chronic lung diseases such as COPD, ILD, asthma, and bronchiectasis (30). However, it is difficult for patients with SSP to undergo PFT before the resolution of pneumothorax (5). A chest tube is usually placed in the pleural space during the treatment of pneumothorax. The chest tube makes it harder for the patients to perform inspiration and expiration because of pleuritic chest pain or discomfort (31). Therefore, previous studies used the data obtained from PFT performed before the occurrence of pneumothorax $(2,19)$. However, there is no mention of the time interval between the assessment of PFT and development of pneumothorax. Therefore, it is not clear whether the PFT results reflect the lung function at the time of the event. In contrast, the present study performed PFT after the resolution of pneumothorax. Fortynine patients underwent PFT within 6 months after the resolution of pneumothorax. This study established and confirmed the association between reduced $\mathrm{FEV}_{1}$ and the recurrence of pneumothorax in patients with PE.

The current study has certain limitations. Primarily, it was based on retrospective data, which could have led to a selection bias. The follow-up protocol used for the prevention of pneumothorax was not standardized and some patients did not undertake PFT or were lost to follow- 
Table 4 Prognostic factors related to recurrence

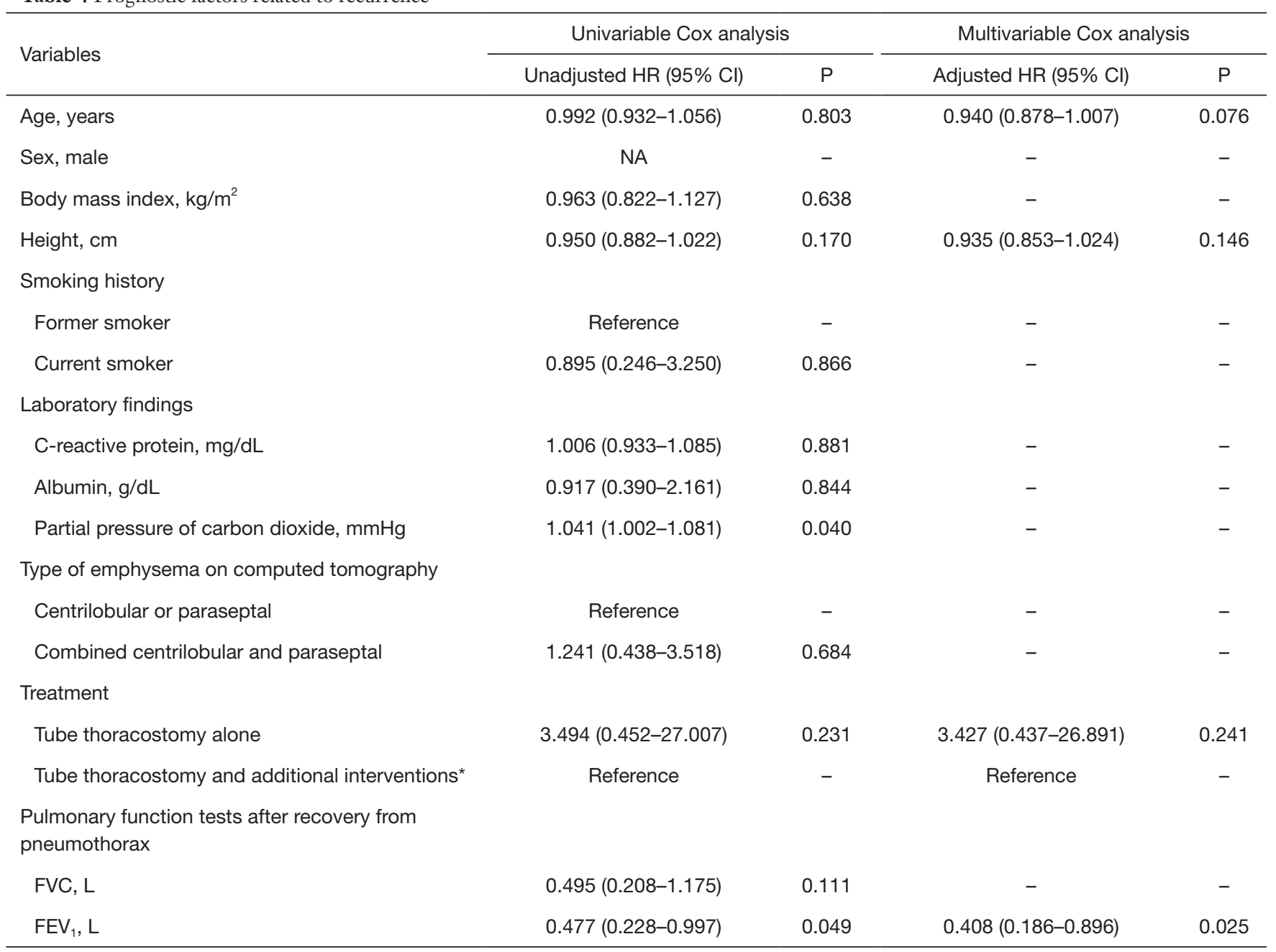

Data are presented as hazard ratio (HR) with 95\% confidence intervals (Cl). *, additional interventions refer to chemical pleurodesis. HR, hazard ratio; $\mathrm{Cl}$, confidential interval; FVC, forced vital capacity; $\mathrm{FEV}_{1}$, forced expiratory volume in 1 second.

up. Therefore, in the current study, the recurrence rate of pneumothorax might have been underestimated. Moreover, the study was performed in a single institution and involved a limited number of subjects. Furthermore, the treatment for pneumothorax was decided by the attending physician, owing to the fact that no standardized treatment protocols have been established for the patients with underlying lung disease and suboptimal lung function.

In conclusion, the overall recurrence rate of pneumothorax was $30.6 \%$ in patients with $\mathrm{PE}$, and the proportion of patients with reduced $\mathrm{FEV}_{1}$ was observed to be significantly higher in the recurrence group. The results of the present study suggest that performing PFT, after the resolution of pneumothorax, could be a useful in the prediction of the recurrence of pneumothorax in patients with PE and pneumothorax.

\section{Acknowledgments}

Funding: None.

\section{Footnote}

Reporting Checklist: The authors have completed the STROBE reporting checklist. Available at http://dx.doi. org/10.21037/jtd-20-1557B

Data Sharing Statement: Available at http://dx.doi. 
org/10.21037/jtd-20-1557B

Peer Review File: Available at http://dx.doi.org/10.21037/jtd20-1557B

Conflicts of Interest: All authors have completed the ICMJE uniform disclosure form (available at http://dx.doi. org/10.21037/jtd-20-1557B). The authors have no conflicts of interest to declare.

Ethical Statement: The authors are accountable for all aspects of the work in ensuring that questions related to the accuracy or integrity of any part of the work are appropriately investigated and resolved. The study was conducted in accordance with the Declaration of Helsinki (as revised in 2013). The study was approved by the Institutional Review Board for Human Research of Yonsei University Wonju Severance Christian Hospital (CR319180). Informed consent was waived of the retrospective nature of the research. Also, only de-identified data extracted from medical records prior to analysis were used in the study.

Open Access Statement: This is an Open Access article distributed in accordance with the Creative Commons Attribution-NonCommercial-NoDerivs 4.0 International License (CC BY-NC-ND 4.0), which permits the noncommercial replication and distribution of the article with the strict proviso that no changes or edits are made and the original work is properly cited (including links to both the formal publication through the relevant DOI and the license). See: https://creativecommons.org/licenses/by-nc-nd/4.0/.

\section{References}

1. Noppen M, De Keukeleire T. Pneumothorax. Respiration 2008;76:121-7.

2. Lee P, Yap WS, Pek WY, et al. An Audit of medical thoracoscopy and talc poudrage for pneumothorax prevention in advanced COPD. Chest 2004;125:1315-20.

3. Sahn SA, Heffner JE. Spontaneous pneumothorax. N Engl J Med 2000;342:868-74.

4. Iga N, Nishi H, Fujimoto N, et al. Clinical features of secondary spontaneous pneumothorax complicated with silicosis. Respir Investig 2018;56:144-9.

5. Shaikhrezai K, Thompson AI, Parkin C, et al. Videoassisted thoracoscopic surgery management of spontaneous pneumothorax--long-term results. Eur J Cardiothorac
Surg 2011;40:120-3.

6. Weissberg D, Refaely Y. Pneumothorax: experience with 1,199 patients. Chest 2000;117:1279-85.

7. Hallifax RJ, Goldacre R, Landray MJ, et al. Trends in the Incidence and Recurrence of Inpatient-Treated Spontaneous Pneumothorax, 1968-2016. JAMA 2018;320:1471-80.

8. Hallifax R, Janssen JP. Pneumothorax-Time for New Guidelines? Semin Respir Crit Care Med 2019;40:314-22.

9. Lippert HL, Lund O, Blegvad S, et al. Independent risk factors for cumulative recurrence rate after first spontaneous pneumothorax. Eur Respir J 1991;4:324-31.

10. Guo Y, Xie C, Rodriguez RM, et al. Factors related to recurrence of spontaneous pneumothorax. Respirology 2005;10:378-84.

11. MacDuff A, Arnold A, Harvey J. Management of spontaneous pneumothorax: British Thoracic Society Pleural Disease Guideline 2010. Thorax 2010;65 Suppl 2:ii18-31.

12. Bobbio A, Chetta A, Carbognani P, et al. Changes in pulmonary function test and cardio-pulmonary exercise capacity in COPD patients after lobar pulmonary resection. Eur J Cardiothorac Surg 2005;28:754-8.

13. Bense L, Eklund G, Wiman LG. Smoking and the increased risk of contracting spontaneous pneumothorax. Chest 1987;92:1009-12.

14. Vogelmeier CF, Criner GJ, Martinez FJ, et al. Global Strategy for the Diagnosis, Management, and Prevention of Chronic Obstructive Lung Disease 2017 Report. GOLD Executive Summary. Am J Respir Crit Care Med 2017;195:557-82.

15. Snider GL, Kleinerman J, Thurlbeck WM, et al. The definition of emphysema. Report of a National Heart, Lung, and Blood Institute, Division of Lung Diseases workshop. Am Rev Respir Dis 1985;132:182-5.

16. Hansell DM, Bankier AA, MacMahon H, et al. Fleischner Society: glossary of terms for thoracic imaging. Radiology 2008;246:697-722.

17. Ruppert AM, Sroussi D, Khallil A, et al. Detection of secondary causes of spontaneous pneumothorax: Comparison between computed tomography and chest X-ray. Diagn Interv Imaging 2020;101:217-24.

18. Sevinc S, Kaya SO, Akcam TI, et al. Prolonged air leakage in secondary spontaneous pneumothorax: is proportion of emphysema important? Clin Respir J 2017;11:833-8.

19. Hobbs BD, Foreman MG, Bowler R, et al. Pneumothorax risk factors in smokers with and without chronic obstructive pulmonary disease. Ann Am Thorac Soc 
2014;11:1387-94.

20. Nam SH, Kim KW, Kim SW, et al. Fate of spontaneous pneumothorax from middle to old age: how to overcome an irritating recurrence? J Thorac Dis 2019;11:4782-9.

21. Smith BM, Austin JH, Newell JD Jr, et al. Pulmonary emphysema subtypes on computed tomography: the MESA COPD study. Am J Med 2014;127:94.e7-23.

22. Baumann MH, Strange C, Heffner JE, et al. Management of spontaneous pneumothorax: an American College of Chest Physicians Delphi consensus statement. Chest 2001;119:590-602.

23. Graham BL, Steenbruggen I, Miller MR, et al. Standardization of Spirometry 2019 Update. An Official American Thoracic Society and European Respiratory Society Technical Statement. Am J Respir Crit Care Med 2019;200:e70-88.

24. Pellegrino R, Viegi G, Brusasco V, et al. Interpretative strategies for lung function tests. Eur Respir J 2005;26:948-68.

25. Choi HS, Park YB, Yoon HK, et al. Validation of Previous Spirometric Reference Equations and New Equations. J Korean Med Sci 2019;34:e304.

Cite this article as: Shin B, Kim SB, Kim CW, Park IH, Lee WY, Byun CS. Risk factors related to the recurrence of pneumothorax in patients with emphysema. J Thorac Dis 2020;12(10):5802-5810. doi: 10.21037/jtd-20-1557B
26. Matsuoka K, Kuroda A, Kang A, et al. Surgical results of video-assisted thoracic surgery and risk factors for prolonged hospitalization for secondary pneumothorax in elderly patients. Ann Thorac Cardiovasc Surg 2013;19:18-23.

27. Nishimoto K, Fujisawa T, Yoshimura K, et al. The prognostic significance of pneumothorax in patients with idiopathic pulmonary fibrosis. Respirology 2018;23:519-25.

28. Watanabe T, Fukai I, Okuda K, et al. Talc pleurodesis for secondary pneumothorax in elderly patients with persistent air leak. J Thorac Dis 2019;11:171-6.

29. Isaka M, Asai K, Urabe N. Surgery for secondary spontaneous pneumothorax: risk factors for recurrence and morbidity. Interact Cardiovasc Thorac Surg 2013;17:247-52.

30. Sim YS, Lee JH, Lee WY, et al. Spirometry and Bronchodilator Test. Tuberc Respir Dis (Seoul) 2017;80:105-12.

31. Luketich JD, Kiss M, Hershey J, et al. Chest tube insertion: a prospective evaluation of pain management. Clin J Pain 1998;14:152-4. 


\section{Supplementary}

Table S1 Pulmonary function tests after recovery from pneumothorax

\begin{tabular}{lccc}
\hline Variables & Recurrence $(\mathrm{n}=15)$ & Ipsilateral $(\mathrm{n}=8)$ & Contralateral $(\mathrm{n}=7)$ \\
\hline FVC, L & $2.97(2.56-3.31)$ & $2.85(2.55-3.17)$ & $3.11(2.56-3.36)$ \\
FVC, \% predicted & $90[74-96]$ & $89[76-92]$ & $95[70-97]$ \\
FEV $_{1}, \mathrm{~L}$ & $1.20(0.91-2.01)$ & $1.05(0.88-1.55)$ & $2.01(1.13-2.41)$ \\
$\mathrm{FEV}_{1}, \%$ predicted & $59[39-97]$ & $55[33-63]$ & $97[53-100]$ \\
FEV 1 /FVC & $0.51(0.32-0.65)$ & $0.39(0.29-0.55)$ & $0.65(0.51-0.72)$ \\
FEV 1 /FVC $<0.7$ & $12(80.0)$ & $8(100)$ & $4(57.1)$ \\
\hline
\end{tabular}

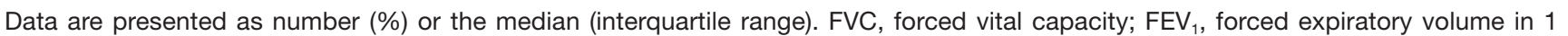
second.

Table S2 Baseline characteristics

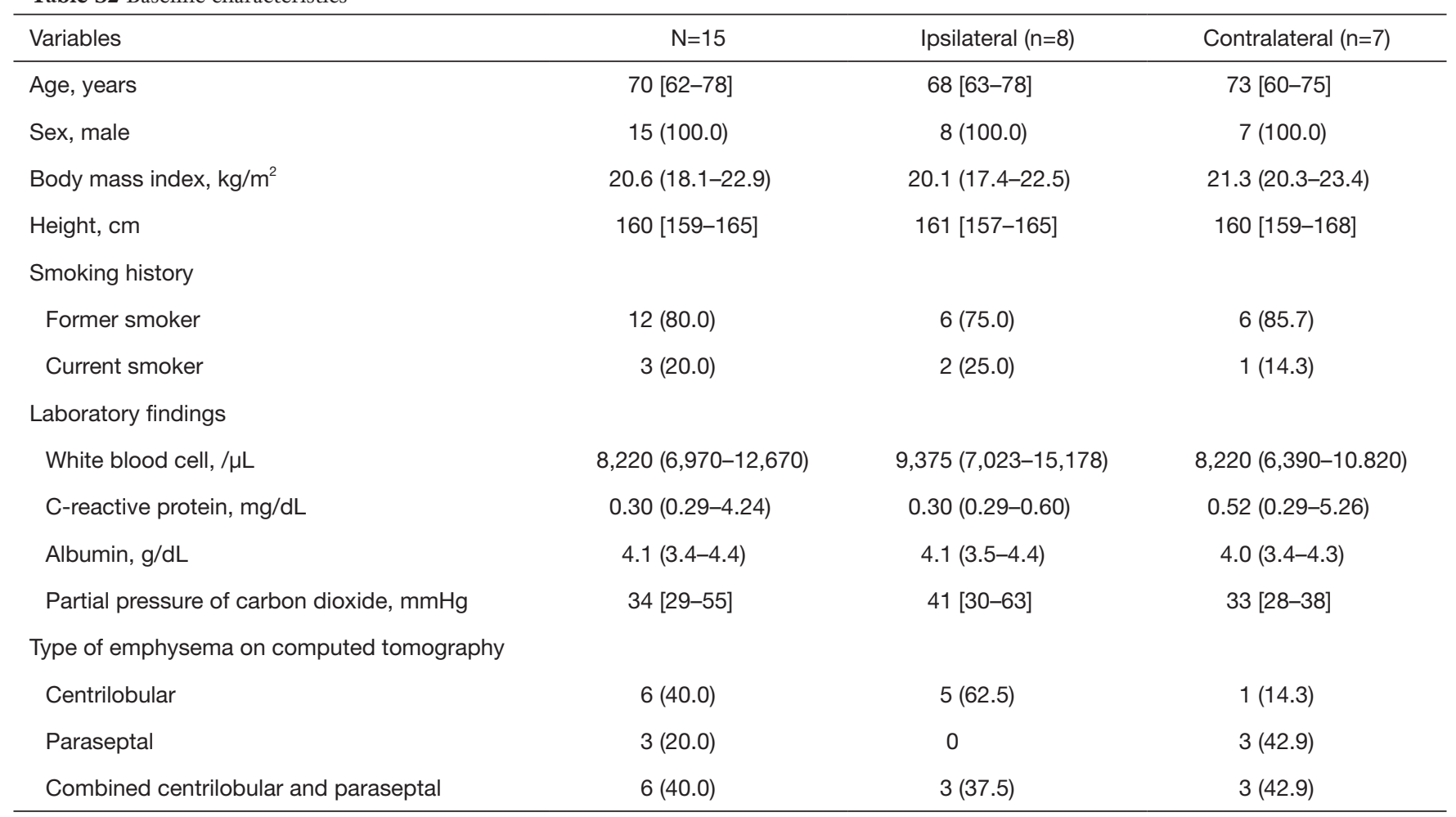

Data are presented as number (\%) or the median (interquartile range). 